\title{
Cooled Infrared Dichroic Beamsplitters and Filters for the MIRI Spectrometer and Imager (5-29 $\mu \mathrm{m})$
}

Book or Report Section

Accepted Version

Hawkins, G. J., Sherwood, R. E. and Barrett, B. M. (2007) Cooled Infrared Dichroic Beamsplitters and Filters for the MIRI Spectrometer and Imager (5-29um). In: Optical Interference Coatings, OSA Technical Digest (CD). Optical Society of America. ISBN 1557528411 Available at http://centaur.reading.ac.uk/977/

It is advisable to refer to the publisher's version if you intend to cite from the work. See Guidance on citing.

Published version at: http://www.opticsinfobase.org/abstract.cfm?URI=OIC-2007-WDPDP1

Publisher: Optical Society of America

Publisher statement: This paper was published in Optical Interference Coatings, OSA Technical Digest (CD) paper WDPDP1 and is made available as an electronic reprint with the permission of OSA. The paper can be found at the following URL on the OSA website: http://www.opticsinfobase.org/abstract.cfm? $U R I=O I C-2007-W D P D P 1$. Systematic or multiple reproduction or distribution to 
multiple locations via electronic or other means is prohibited and is subject to penalties under law.

All outputs in CentAUR are protected by Intellectual Property Rights law, including copyright law. Copyright and IPR is retained by the creators or other copyright holders. Terms and conditions for use of this material are defined in the End User Agreement.

\section{www.reading.ac.uk/centaur}

\section{CentAUR}

Central Archive at the University of Reading

Reading's research outputs online 


\title{
Cooled Infrared Dichroic Beamsplitters and Filters for the MIRI Spectrometer and Imager $(5-29 \mu \mathrm{m})$
}

\author{
G.J. Hawkins, R.E. Sherwood, B.M. Barrett \\ The University of Reading, Infrared Multilayer Laboratory, School of Systems Engineering, \\ Whiteknights, Reading, Berkshire, RG6 6 AY, UK \\ e-mail: g.j.hawkins@reading.ac.uk
}

\begin{abstract}
The spectral design and fabrication of cooled $(7 \mathrm{~K})$ mid-infrared dichroic beamsplitters and bandpass filter coatings for the MIRI spectrometer and imager are described. Design methods to achieve the spectral performance and coating materials are discussed.

(C) Optical Society of America

OCIS Codes: $310.0310,310.1620,310.1860$
\end{abstract}

\section{0}

\section{Introduction}

The Mid-Infrared Instrument (MIRI ${ }^{[1]}$ is a cooled $(7 \mathrm{~K})$ thermal-infrared imager and medium resolution spectrometer being developed for launch on the JWST (James Webb Space Telescope) in 2013 by an international consortium of European partners sponsored by the European Space Agency, the Jet Propulsion Laboratory and an international science team. The University of Reading is responsible for the design and fabrication of the long-wave pass dichroic beamsplitters in the spectrometer sub-system $(5-29 \mu \mathrm{m})$ together with a set of bandpass filters $(13-29 \mu \mathrm{m})$, and chronographic filter $(23 \mu \mathrm{m})$ for the wide-field imaging camera.

The spectrometer sub-system in Figure 1 contains four channels (1-4x) which spectrally multiplex the incoming radiation. The incident ray bundles are divided between channels using an arrangement of three sets of dichroic beamsplitters (1-3) combined in series along the optical trains. The four channels, with overlapping wavelengths are each split into three wavelength ranges (a-c) to provide the resolving power on the available (Si:As) detector arrays. The splitting of the wavelengths within each channel in Table 1 is achieved using three separate sets of dichroics and diffraction gratings, mounted on two wheels. The spectral design specifications ${ }^{[2]}$ of the dichroic beamsplitters required compliance of reflective and transmissive bands at a temperature of $7 \mathrm{~K}$ in an illuminated converging cone of $f / 37$ and tilt angle of 10 degrees. The coatings were deposited on to $21 \mathrm{~mm}$ diameter $\mathrm{x} 5.0 \mathrm{~mm}$ thick optical grade CVD cadmium telluride (CdTe) substrates to provide the longest non-hygroscopic passband transparency $(\sim 30 \mu \mathrm{m})$ and durability. Short wavelength blocking required interference rejection levels of $<10^{-4}$ for the cumulative optical train for all wavelengths shorter than the limit placed on the reflective shortest wavelength band.

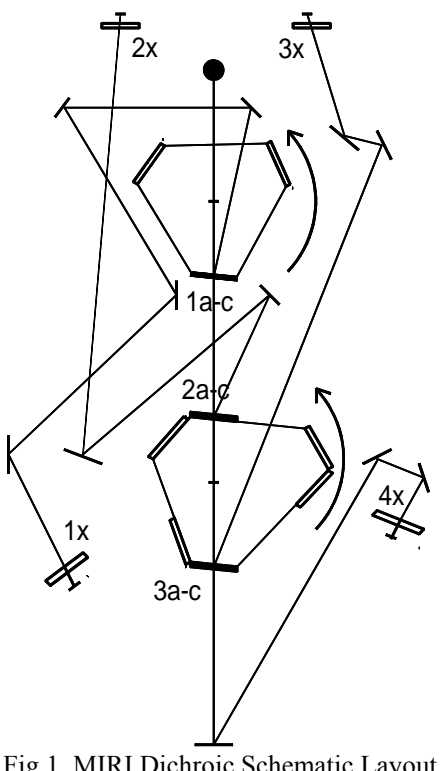

Table 1. Reflection \& Transmission Bands for MIRI Dichroic Beamsplitters at $7 \mathrm{~K}$

\begin{tabular}{|c|c|c|}
\hline Dichroic & $\begin{array}{c}\text { Reflection Band } \\
(\mu \mathrm{m})\end{array}$ & $\begin{array}{c}\text { Transmission } \\
\text { Band }(\mu \mathrm{m})\end{array}$ \\
\hline 1a & $4.86-5.81$ & $7.43-21.16$ \\
\hline $1 \mathrm{~b}$ & $5.61-6.71$ & $8.59-24.66$ \\
\hline 1c & $6.48-7.74$ & $9.91-28.75$ \\
\hline 2a & $7.43-8.88$ & $11.44-21.16$ \\
\hline 2b & $8.59-10.26$ & $13.22-24.66$ \\
\hline 2c & $9.91-11.84$ & $15.27-28.75$ \\
\hline 3a & $11.44-13.64$ & $17.53-21.16$ \\
\hline 3b & $13.22-15.76$ & $20.43-24.66$ \\
\hline 3c & $15.27-18.20$ & $23.82-28.75$ \\
\hline
\end{tabular}

Fig 1. MIRI Dichroic Schematic Layout 
To achieve a high image performance, the optical metrology and surface quality required transmitted and reflective wavefront errors (WFE) and parallelism to be $<\lambda / 10$ peak-valley at the shortest wavelength transmitted (or reflected) bands, and maximum wedge angle of $<1$ arcmin. To maintain these tolerances, careful consideration of disposition of multilayer designs was necessary to minimize thickness differences between surfaces and equalize stress to avoid distortion. The environmental requirements included durability to survive cryogenic cooling with no evidence of stress fracturing, and compliance to the general provisions of MIL-F-48616 including repeated thermal cycling between $300 \mathrm{~K}$ and $77 \mathrm{~K}$. The highest deposition coating quality, possessing a minimum of scattering sites caused by surface roughness or spatter seeds was required together with spectral stability for an operational period of 10 years.

\subsection{Coating Design and Materials}

The multilayer design approach to achieve the high broad transparency and reflectivity bands was by refinement of Tchebysheff equi-ripple ${ }^{[3]}$ long-wave pass edge filters. With a high index contrast, these designs possess a wide shortwave rejection stop-band, providing high reflectivity which together with appropriate thickness and index matching, give wide passband transparency. A series of broadband antireflection coatings $(4.5-30 \mu \mathrm{m})$ were further developed to minimize reflections from the rear surface. It was necessary to encompass the complete range of reflective and transmissive wavelengths of each channel to minimize ghosting artifacts resulting from rear surface reflections. Each layer was of unique non-quarterwave fractional thickness to minimize passband ripple amplitude.

Layer materials available in the mid-infrared that can be deposited on CdTe with high durability and optical performance are limited, particularly where transparency is required at far-infrared wavelengths $(>20 \mu \mathrm{m})$. Lead Telluride $(\mathrm{PbTe})$ and/or Germanium (Ge) alternated with II-VI group dielectrics dominate the materials selection due to their high molecular weights and wide electronic band-gap. PbTe (H-layer, $n \sim 5.85)$ in combination with $\mathrm{CdSe}(\mathrm{L}-$ layer, $n \sim 2.34$ ) was selected as the most appropriate durable layer material combination for the longer wavelength passbands (1c-3c) particularly as the high refractive index contrast together with long-wave semiconductor absorption edge assist to minimize the structural thickness and number of layers. However, as the 1a-1b dichroics required shortwave reflection bands within the $\mathrm{PbTe}$ semiconductor absorption region, a set of $\mathrm{Ge} / \mathrm{CdSe}$ broad passband multilayers were developed for this region. The rear surface antireflection coating comprised alternate layers of $\mathrm{Ge} / \mathrm{CdSe}$ for dichroics $1 \mathrm{a} \& \mathrm{~b}$ and $\mathrm{PbTe} / \mathrm{CdSe}$ for $1 \mathrm{c}-3 \mathrm{c}$. This structure was overcoated with a low-index $(n \sim 1.35) \mathrm{BaF}_{2}$ layer to assist the refractive index matching and provide a broad antireflection bandwidth response in thin film form. The $\mathrm{BaF}_{2}$ layer was environmentally protected within the multilayer by additional CdSe overcoating.

\subsection{Fabrication and Measurements}

Fabrication of the dichroics (Figs. 2-4) and imager filters (Fig. 6) was performed by vacuum deposition using a modified Balzers BA510 evaporator fitted with tooling of our historic in-house design. This tooling is unique comprising stationary substrates and rotating thermal evaporation sources to provide a temperature stable platform with in situ optical reflectance monitoring previously described elsewhere ${ }^{[4]}$. Spectral measurements were performed in a PE2000 Optica FTIR spectrometer with cooled transmission measured through a helium cryostat fitted with KRS-5 windows. Reflectivity measurements at RT were verified at $7 \mathrm{~K}$ by spectral modeling using optical constants.

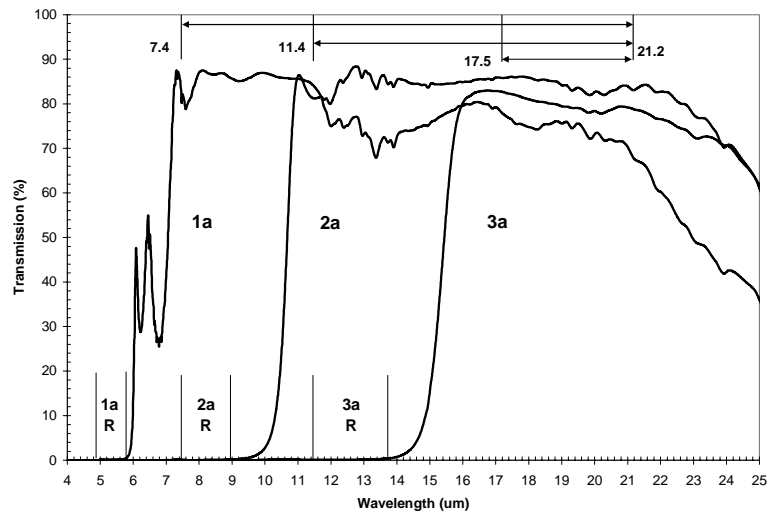

Fig 2. MIRI 1-3a Dichroic filter channel overlay at 7K

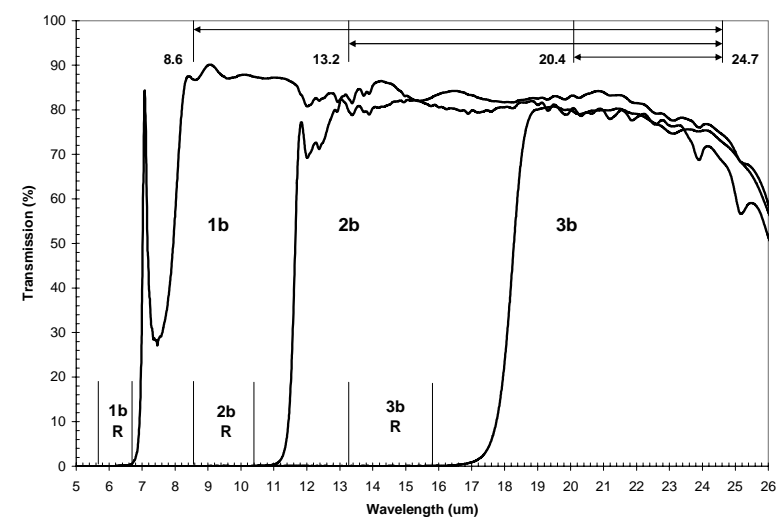

Fig 3. MIRI 1-3b Dichroic filter channel overlay at 7K 


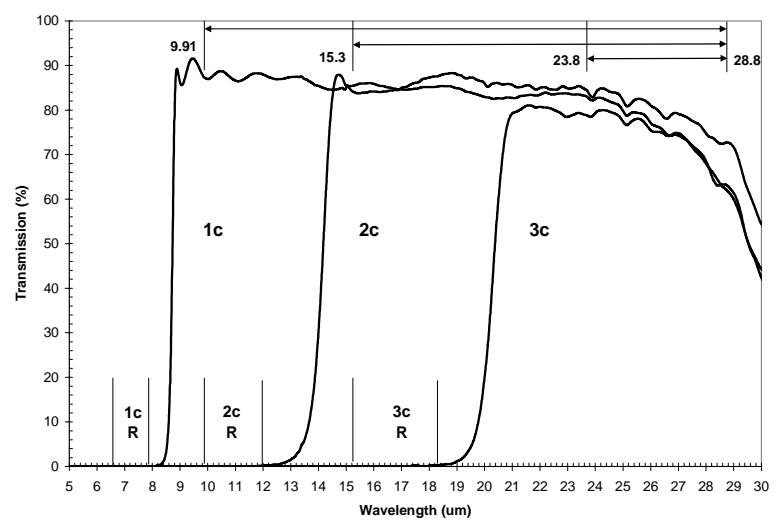

Fig 4. MIRI 1-3c Dichroic filter channel overlay at 7K

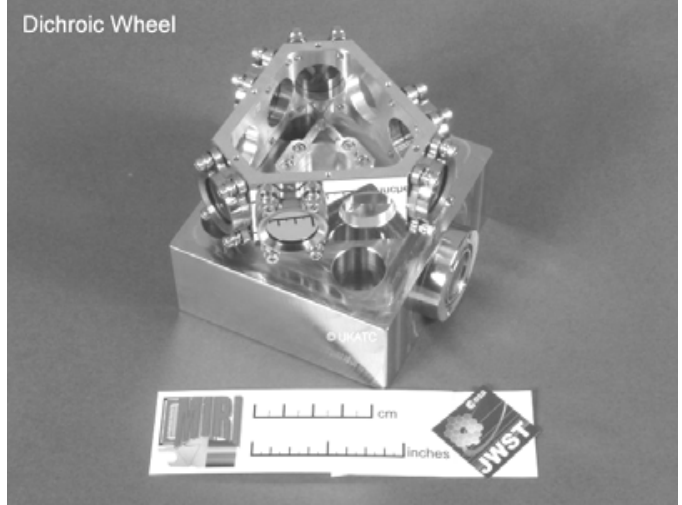

Fig 5. MIRI Dichroic beamsplitter wheel construction

\subsection{MIRI Imager Bandpass Filters}

Bandpass filters for the MIRIM imager module (IM06-08) comprised combinations of long and shortwave pass edge filters fabricated using $\mathrm{PbTe} / \mathrm{CdSe}$ with overlapping rejection stop-bands to provide continuous wavelength blocking. Rejection levels of $<3 \times 10^{-4}$ were required for each channel to coincide with the photo-absorption edge of PbTe at $\sim 5.5 \mu \mathrm{m}$, and multiphonon absorption of ZnSe (IM06), and CdTe (IM07-08) at 22 $\mu \mathrm{m}$ and $32.5 \mu \mathrm{m}$ respectively. Filter IM09 comprised a longwave pass blocking filter with $29 \mu \mathrm{m}$ cut-off wavelength described by the detector response.

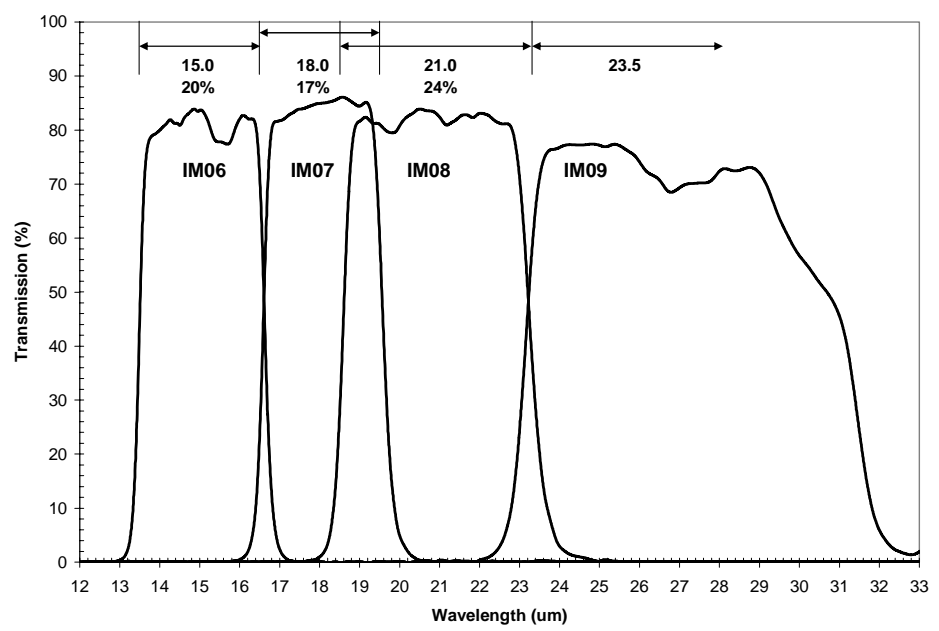

5.0 Conclusions

Fig 6. MIRI Imager filter measurements at $7 \mathrm{~K}$

The spectral design, manufacture and testing of the JWST MIRI dichroic beamsplitters and filters have been described, together with the choice of substrate and coating materials. The completed filters have been fully accepted in compliance with the specified instrument requirements and are being assembled for integration and launch.

\section{Acknowledgements}

The authors thank colleagues at the AlbaNova University Centre - Stockholm Centre for Physics and Astronomy, and Dublin Institute of Advanced Studies, School of Cosmic Physics whose support is gratefully acknowledged.

1. G.S. Wright, F.Bortoletto, C.F. Bruce, Jr., E.F. van Dishoeck, A.R. Karnik, P.O. Lagage, M.E. Larson, D.Lemke, G. Oloffson, E.A. Miller, T.F. Henning, S. Heys, T. Ray, J. Rodriguez, E. Serabyn, I. Walters: "NGST MIRI instrument” SPIE 4850, pp. 493-503 (2003).

2. James Webb Space Telescope (JWST) Mid-Infrared Instrument (MIRI): "Requirements specification for the MIRI spectrometer filters", MIRI-RS-00004-ATC Issue E (2004) \& MIRI-RS-0002-ODP (2005), otherwise unpublished.

3. MJ Wells, G J Hawkins, G Olofsson: "The design and fabrication of multiple dichroic beamsplitters for the MIRI spectrometer (4.829 um)", Proc SPIE Vol. 5487, pp 794-803 (2004)

4. C. Evans, R. Hunneman, J. Seeley, A. Whatley: "Filters for v $v_{2}$ band of $\mathrm{CO}_{2}$ : monitoring and control of layer deposition", Applied Optics, Vol. 15, No. 11, pp. 2736-2745 (1976). 